\title{
PRODUTO DE PANIFICAÇÃO COM FARINHA DE ARROZ ENRIQUECIDA COM FARINHA DE ORA-PRO-NÓBIS (Pereskia aculeata)
}

\author{
BENETOLE, Bianca Martins ${ }^{1}$ \\ GOMES, Winston Pinheiro Claro ${ }^{2}$ \\ CAMPOS, Stéfane Verde de ${ }^{3}$ \\ BORTOLETO, Gisele Gonçalves ${ }^{4}$ \\ GUTIERREZ Erika Maria Roel ${ }^{5}$
}

\begin{abstract}
Recebido em: 2020.06 .23
Aprovado em: 2020.09 .29

ISSUE DOI: $10.3738 / 1982.2278 .3809$

RESUMO: A ora-pro-nóbis é uma Planta Alimentícia Não Convencional (PANC), que possui alto teor de cálcio e fibras, atividades associadas ao sistema imune, propriedades de absorção de água e capacidade funcional de redução de colesterol ruim (LDL). Assim, o objetivo principal foi criar um produto de panificação fortificado, utilizando-se da farinha de arroz enriquecida com farinha de ora-pro-nóbis, no caso, um bolo. As análises do produto final foram realizadas de acordo com Instituto Adolfo Lutz (2008), Asp et al. (1983) e Moretto et al. (2002). As gorduras saturadas e trans foram calculadas conforme TACO (2011) e Marinelli (2016). Criou-se um produto com aumento de $4 \%$ nos teores de cálcio e de fibra alimentar e com valor inferior a $1 \%$ de gorduras totais, que apresentou $9 \mathrm{kcal}$ a menos do que o produto sem a farinha de ora-pro-nóbis. A PCA auxiliou no entendimento das diferenças e similaridades dos produtos com e sem a farinha de ora-pro-nóbis, levando a concluir que o bolo enriquecido com a farinha de ora-pro-nóbis apresenta características nutricionais superiores ao bolo elaborado apenas com a farinha de arroz. É importante destacar que outros produtos inovadores a partir da ora-pro-nóbis podem ser elaborados, visto as suas interessantes características nutricionais e terapêuticas.
\end{abstract}

Palavras-chave: Planta Alimentícia Não Convencional. Composição Centesimal. Quimiometria.

\section{BAKERY PRODUCT WITH RICE FLOUR ENRICHED WITH BARBADOS GOOSEBERRY (Pereskia aculeata) FLOUR}

\begin{abstract}
SUMMARY: The Barbados gooseberry is a Non-Conventional Food Plant (PANC), which has a high content of calcium and fibers, activities associated with the immune system, water absorption properties and functional capacity to reduce bad cholesterol (LDL). Therefore, the main objective was to create a gluten-free bakery product, using rice flour enriched with Barbados gooseberry flour, in this case, a cake. The analysis is performed according to Institute Adolfo Lutz (2008), Asp et al. (1983) and Moretto et al. (2002). Saturated and Trans fats were calculated according to TACO (2011) and Marinelli (2016). It was possible to create a product with a $4 \%$ increase in calcium and dietary fiber content and a lower value of $1 \%$ in total fats, and even containing $1.5 \mathrm{~g}$ more carbohydrate in its composition, the cake with Barbados gooseberry presented $9 \mathrm{kcal}$ less than the cake without Barbados gooseberry flour. One can concluded that the cake enriched with Barbados gooseberry flour presents nutritional characteristics superior to the cake made only with rice flour. PCA helped to understand the differences and similarities of products with and without Barbados gooseberry flour, leading us to conclude that the cake enriched with Barbados gooseberry flour presents nutritional characteristics superior to the cake made only with the rice flour. It is important to highlight that others innovative products from Barbados gooseberry may be developed, given their interesting nutritional and therapeutic characteristics.
\end{abstract}

Keywords: Non-Conventional Food Plant. Centesimal composition. Chemometrics.

\footnotetext{
${ }^{1}$ ORCID iD - https://orcid.org/0000-0002-2224-5186. FATEC- Piracicaba.

2 ORCID iD - https://orcid.org/0000-0003-1165-8572

$3 \frac{\text { ORCID iD }}{\text { Ottps://orcid.org/0000-0002-2224-5186 }}$

4 ORCID iD - https://orcid.org/0000-0002-3425-3888

5 ORCID iD $-\underline{\text { https://orcid.org/0000-0003-4432-8297 }}$
} 


\section{INTRODUÇÃO}

Em 2018, mais de 143 milhões de pessoas em 53 países sofreram de insegurança alimentar aguda, necessitando de alimento imediato e nutritivo, além de assistência para obterem meios de subsistência, o que demonstra o quanto segurança alimentar é um desafio global (FAO, 2019).

Neste contexto, um alimento que merece destaque é o arroz, disponível para a população em vários locais do planeta e que, além de possuir um alto teor de proteínas e baixo teor de lipídeos, apresenta características não alergênicas e é rico em sais minerais, vitaminas de complexo B (DORS, PINTO ; BADIALE-FURLONG, 2009; FRANCO et al., 2018).

Outro produto que também merece atenção é a ora-pro-nóbis (Pereskia aculeata), que é uma hortaliça classificada como uma Planta Alimentícia Não Convencional (PANC), com ausência de toxicidade de suas folhas e importantes qualidades nutritivas, como cálcio e alto teor de proteínas e de fibras (ALMEIDA; CORRÊA, 2012; ALMEIDA et al., 2014; OLIVEIRA, 2017). Apresenta em sua composição as arabinogalactanas, com atividades associadas ao sistema imune (SEYFRIED, 2016) e as galactomanas, com propriedades de absorção de água que auxiliam no funcionamento do trato digestivo (TONON et al., 2018). Ainda, contém os fitoesteróis, sitosterol e estigmasterol, que apresentam capacidade funcional de redução das concentrações do colesterol ruim (LDL) (ALMEIDA; CORRÊA, 2012) e os 9 aminoácidos essenciais, a saber, Histidina (0,62 g); Isoleucina (1,13 g); Leucina (2,01 g); Lisina (1,75 g); Metionina (0,35 g); Fenilalanina (1,47 g); Treonina (1,03 g); Triptofano (0,55 g); Valina (1,29 g) (MARINELLI, 2016). Deve ser ressaltado ainda que o consumo da farinha de ora-pro-nóbis, por seres humanos, aumenta a saciedade (VIEIRA et al., 2019).

Além da segurança alimentar, outro grande desafio do mercado, mas de caráter tecnológico, é do desenvolvimento de produtos alimentícios sem glúten que proporcionem qualidade sensorial com potencial para promover melhorias nos cuidados da saúde e, ao mesmo tempo, na qualidade de vida das pessoas com doença celíaca (GALLAGHER, GORMLEY ; ARENDT, 2004; FASANO et al., 2008; NASCIMENTO, 2014).

Assim, a proposta do presente trabalho aborda a elaboração de um produto com uma elevada concentração de proteína de fonte vegetal. Para tal, criou-se um produto de panificação, no caso, um bolo, utilizando-se de farinha de arroz enriquecida com farinha de ora-pro-nóbis. 
Verificou-se a composição centesimal do produto final, com a diferenciação através de ferramentas quimiométricas e elaborou-se um rótulo nutricional.

A mistura das farinhas de arroz e de ora-pro-nóbis deveria ser levada em consideração como uma interessante alternativa na busca de novos produtos, com alta concentração de proteína e que não contém glúten.

\section{METODOLOGIA}

As amostras de ora-pro-nóbis ( $P$. aculeata) foram coletadas de plantas matrizes mantidas na Faculdade de Tecnologia de Piracicaba Deputado Roque Trevisan, localizada na cidade de Piracicaba/SP durante o primeiro semestre de 2019. A seleção de galhos foi aleatória, tendo todas as suas folhas retiradas desde os brotos até as mais velhas.

Após a coleta, as folhas foram levadas até o Laboratório de Alimentos, lavadas com água e imersas em solução de 200 ppm de hipoclorito de sódio litro ${ }^{-1}$ de água durante 15 minutos, para então serem lavadas novamente em água corrente, guardadas em sacos de polipropileno, etiquetadas e armazenadas em freezer convencional.

Depois de bem congeladas as folhas foram submetidas ao processo de liofilização, utilizando-se o liofilizador de modelo Alpha 2-4 LDplus de laboratório com capacidade de 4 $\mathrm{kg}$ de gelo, que foi programado para uma pressão de 0,12 mbar (milésimos de bar) e uma temperatura de $-87^{\circ} \mathrm{C}$ (Celsius) pelo tempo de 24 a 48 horas.

Após a liofilização, o material foi triturado em liquidificador e adicionado, em termos de $15 \%$, à farinha de arroz com a finalidade de enriquecimento da farinha e do produto final. A farinha de arroz enriquecida com ora-pro-nóbis foi utilizada no preparo de um bolo recheado com ganache de chocolate e cobertura de açúcar de confeiteiro, conforme a receita apresentada na Tabela 1 e Figura 1, que foi pré-seco em estufa, por 24 horas a temperatura de $60{ }^{\circ} \mathrm{C}$, macerado e utilizado nas análises posteriores realizadas em triplicata. Além disso realizou-se o preparo de um bolo nos mesmos moldes, conforme a Tabela 1, para fins comparativos entre as composições centesimais. 
Tabela 1 - Receita do bolo enriquecido com a farinha de ora-pro-nóbis e do bolo sem o enriquecimento.

\begin{tabular}{c|c|c}
\hline Ingredientes & $\begin{array}{c}\text { Bolo com farinha de } \\
\text { ora-pro-nóbis (g) }\end{array}$ & $\begin{array}{c}\text { Bolo sem farinha de } \\
\text { ora-pro-nóbis (g) }\end{array}$ \\
\hline Farinha de arroz & 85,0 & 100,0 \\
\hline Farinha de ora-pro-nóbis & 15,0 & - \\
\hline Margarina sem sal & 75,0 & 75,0 \\
\hline Ovo & 139,0 & 139,0 \\
\hline Baunilha & 10,0 & 10,0 \\
\hline Fermento químico & 12,0 & 12,0 \\
\hline
\end{tabular}

Fonte: Elaborado pelos Autores.

Figura 1 - Bolo de farinha de arroz enriquecida com a farinha de ora-pro-nóbis

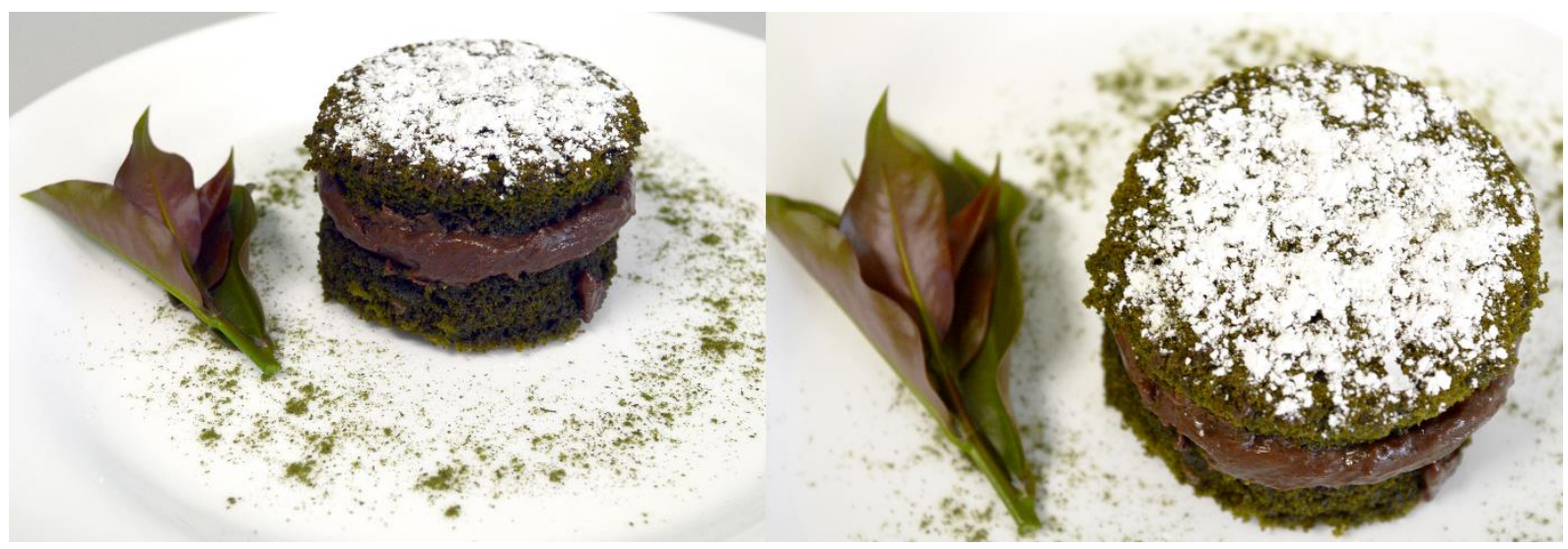

Fonte: Elaborado pelos Autores.

O recheio utilizado para ambos os bolos, são iguais, onde se utilizou 202,0 g de chocolate ao leite, 134,0 g de creme de leite e para cobertura se utilizou 9,4 g de açúcar de confeiteiro, também em ambos os bolos.

A umidade do bolo foi determinada por dessecação em estufa a $105^{\circ} \mathrm{C}$ até peso constante. O extrato etéreo foi determinado utilizando-se extrator contínuo tipo Soxhlet. A proteína bruta foi dosada pelo método Kjeldahl, utilizando o fator de conversão 6,25 ( $\mathrm{N} \mathrm{x}$ $6,25)$. As cinzas foram obtidas pela incineração $\left(550^{\circ} \mathrm{C}\right)$ em forno tipo mufla. O cloreto de sódio foi dosado diretamente pelo método argentométrico de Mohr, sendo todas as análises realizadas conforme descrito por Instituto Adolfo Lutz (2008). A fibra alimentar foi quantificada pelo método enzimático, descrito por Asp et al. (1983). Os carboidratos 
disponíveis foram determinados pela diferença, em matéria seca, entre 100 e a soma de lipídeos, cinzas, proteínas e fibra alimentar total.

Enquanto o teor do mineral cálcio $(\mathrm{Ca})$ foi determinado conforme descrito por Moretto et al. (2002) e os teores de gordura saturada e gordura trans presentes no rótulo nutricional foram calculados a partir de informações da tabela TACO, (2011) e de Marinelli (2016).

A relação da composição centesimal do produto com e sem a farinha de ora-pro-nóbis foi investigada através da Análise de Componentes Principais (ACP) utilizando o software Past 4.0 (HAMMER, HARPER ; RYAN, 2001). A ACP é uma técnica estatística de análise multivariada cuja intenção é a redução da massa de dados com menor perda possível da informação, sendo que ela agrupa os indivíduos de uma população segundo a variação de suas características (HONGYU, SANDANIELO ; OLIVEIRA JUNIOR, 2016).

As variáveis químicas consideradas serão os teores finais dos compostos determinados.

\section{RESULTADO E DISCUSSÃO}

Após obter os dados através da análise centesimal e com auxílio do software Microsoft Excel®, foram calculados e os teores em base úmida de umidade, cinza, proteína, óleos, fibras, carboidratos, sódio e cálcio do bolo enriquecido com ora-pro-nóbis e do bolo contendo apenas farinha de arroz, apresentados na Tabela 2.

Tabela 2 - Teores da composição centesimal em base úmida de cada tipo de bolo.

\begin{tabular}{c|c|c}
\hline Composição & $\begin{array}{c}\text { Bolo com farinha de } \\
\text { ora-pro-nóbis }\end{array}$ & $\begin{array}{c}\text { Bolo sem farinha } \\
\text { de ora-pro-nóbis }\end{array}$ \\
\hline Umidade (\%) & $28,24 \pm 0,096$ & $27,85 \pm 0,151$ \\
\hline Cinzas (\%) & $2,33 \pm 0,042$ & $1,87 \pm 0,033$ \\
\hline Extrato Etéreo (\%) & $22,92 \pm 1,300$ & $24,22 \pm 0,414$ \\
\hline Proteínas (\%) & $7,83 \pm 0,718$ & $7,43 \pm 0,543$ \\
\hline Fibra Alimentar Total (\%) & 7,80 & 5,27 \\
\hline Fibras solúveis (\%) & $2,04 \pm 0,469$ & $1,22 \pm 0,224$ \\
\hline Fibras insolúveis (\%) & $5,76 \pm 0,440$ & $4,05 \pm 0,181$ \\
\hline Carboidratos disponíveis (\%) & 13,92 & 17,79 \\
\hline Cálcio (mg/100g) & $167,85 \pm 5,653$ & $65,81 \pm 16,952$ \\
\hline Sódio (mg/100g) & $27,31 \pm 7,350$ & $21,57 \pm 7,345$ \\
\hline
\end{tabular}

Fonte: Elaborado pelos Autores.

Também foram calculados os valores energéticos e teores de carboidratos, proteínas, gorduras, fibras alimentares, sódio e cálcio para os rótulos nutricionais elaborado conforme a Agência Nacional de Vigilância Sanitária (BRASIL,2003a, 2003b), tanto do bolo com farinha 
de ora-pro-nóbis quanto no bolo sem farinha de ora-pro-nóbis, apresentados nas Tabelas 3 e 4 , respectivamente.

Tabela 3 - Rótulo nutricional do bolo com farinha de ora-pro-nóbis.

\begin{tabular}{l|c|c}
\hline \multicolumn{3}{c}{$\begin{array}{c}\text { INFORMAÇÃO NUTRICIONAL } \\
\text { Porção de } 40 \mathrm{~g}(1 \mathrm{fatia})\end{array}$} \\
\hline & $\begin{array}{c}\text { Quantidade por } \\
\text { Porção }\end{array}$ & $\% \mathrm{VD}\left(^{*}\right)$ \\
\hline Valor Energético & $154 \mathrm{kcal} \mathrm{e} 643 \mathrm{~kJ}$ & $8 \%$ \\
\hline Carboidratos & $6 \mathrm{~g}$ & $2 \%$ \\
\hline Proteínas & $3 \mathrm{~g}$ & $4 \%$ \\
\hline Gorduras Totais & $9 \mathrm{~g}$ & $17 \%$ \\
\hline Gorduras Saturadas & $6 \mathrm{~g}$ & $(* *)$ \\
\hline Gorduras Trans & $0,1 \mathrm{~g}$ & $12 \%$ \\
\hline Fibra Alimentar & $3 \mathrm{~g}$ & $0 \%$ \\
\hline Sódio & $11 \mathrm{mg}$ & $7 \%$ \\
\hline Cálcio & $67 \mathrm{mg}$ & $\mathrm{g} \%$ \\
\hline
\end{tabular}

* \% Valores Diários de Referência com base em uma dieta de 2000 Kcal ou 8400 KJ. Seus

Valores diários podem ser maiores ou menores dependendo de suas necessidades energéticas. (**) Valores Diários de Referência não estabelecidos.

Lista de Ingredientes: Chocolate ao leite, Ovo, Creme de leite, Farinha de arroz, Margarina,

Farinha de ora-pro-nóbis, Fermento químico, Baunilha e Açúcar de confeiteiro.

Fonte: Elaborado pelos Autores.

Tabela 4 - Rótulo nutricional do bolo sem farinha de ora-pro-nóbis.

\begin{tabular}{|c|c|c|}
\hline \multicolumn{3}{|c|}{$\begin{array}{c}\text { INFORMAÇÃO NUTRICIONAL } \\
\text { Porção de } 40 \text { g (1 fatia) } \\
\end{array}$} \\
\hline & $\begin{array}{l}\text { Quantidade por } \\
\text { Porção }\end{array}$ & $\% \mathrm{VD}(*)$ \\
\hline Valor Energético & $163 \mathrm{kcal} \mathrm{e} 680 \mathrm{~kJ}$ & $8 \%$ \\
\hline Carboidratos & $7 \mathrm{~g}$ & $2 \%$ \\
\hline Proteínas & $3 \mathrm{~g}$ & $4 \%$ \\
\hline Gorduras Totais & $10 \mathrm{~g}$ & $18 \%$ \\
\hline Gorduras Saturadas & $6 \mathrm{~g}$ & $28 \%$ \\
\hline Gorduras Trans & $0,1 \mathrm{~g}$ & $(* *)$ \\
\hline Fibra Alimentar & $2 \mathrm{~g}$ & $8 \%$ \\
\hline Sódio & $9 \mathrm{mg}$ & $0 \%$ \\
\hline Cálcio & $26 \mathrm{mg}$ & $3 \%$ \\
\hline \multicolumn{3}{|c|}{$\begin{array}{l}\text { *\% Valores Diários de Referência com base em uma dieta de } 2000 \text { Kcal ou } 8400 \text { KJ. Seus } \\
\text { Valores diários podem ser maiores ou menores dependendo de suas necessidades energéticas. } \\
(* *) \text { Valores Diários de Referência não estabelecidos. }\end{array}$} \\
\hline \multicolumn{3}{|c|}{$\begin{array}{c}\text { Lista de Ingredientes: Chocolate ao leite, Ovo, Farinha de arroz, Creme de leite, Margarina, } \\
\text { Fermento químico, Baunilha e Açúcar de confeiteiro. }\end{array}$} \\
\hline
\end{tabular}

Fonte: Elaborado pelos Autores. 
A partir das Tabelas 2, 3 e 4, por meio dos resultados das porcentagens dos constituintes nutricionais, foi possível fazer as comparações entre os diferentes bolos formulados. Todas as comparações foram realizadas em relação a base úmida, já que o produto será consumido dessa maneira.

\subsection{Teores de Umidade}

A umidade é um dos fatores que determina o tempo de conservação de um alimento, e a relação que se estabelece é que quanto maior a umidade, mais perecível é o alimento (BOLZAN, 2013). Conforme a Tabela 2, observou-se que o bolo contendo apenas farinha de arroz obteve um teor de $0,39 \%$ a menos de umidade comparado ao bolo contendo a farinha ora-pro-nóbis. Sato et al. (2019), na análise centesimal de massas de macarrão contendo as folhas de ora-pro-nóbis e convencional, observou que a massa convencional também apresentou um teor menor de umidade, no caso, com uma diferença de 1,37\% a menos em comparação com a massa de macarrão formulada com a hortaliça. Esse aumento no teor de umidade se deve ao fato da farinha de ora-pro-nóbis acrescentar maiores teores de fibras aos alimentos, e, considerando que as fibras alimentares possuem grande números de grupos hidroxilas em sua estrutura, isso facilita a formação de ligações de hidrogênio e, por conseguinte, aumenta-se a absorção de água pelo alimento (BORGES et al., 2011). No entanto, um aumento umidade no produto final com teores bem menores que $1 \%$ não deve ser levado como fator relevante para diminuir consideravelmente o shelf-life do produto.

\subsection{Teores de Cinzas}

As cinzas representam os resíduos inorgânicos, ou seja, os minerais contidos nas amostras (VIZZOTTO et al., 2010). Rocha et al. (2008) menciona que os minerais têm papel importante na constituição do organismo e regulam o metabolismo de algumas enzimas. Além disso, a deficiência de um ou mais minerais pode resultar em grandes distúrbios no organismo como, por exemplo, a osteoporose.

Neste trabalho observou-se que o bolo com farinha de ora-pro-nóbis teve um aumento do teor de cinzas de $0,45 \%$, em relação ao produto sem a farinha de ora-pro-nóbis, o que leva a concluir que houve aumento nos teores dos minerais no produto estudado. Zem (2017), em seu trabalho, obteve um aumento de $0,07 \%$ para o cupcake e $1,41 \%$ para o macarrão, quando estes produtos tiveram a adição de $30 \%$ de farinha de ora-pro-nóbis em sua formulação. Esse 
aumento das cinzas se deve ao fato de que em todos os produtos formulados foram utilizadas as folhas da ora-pro-nóbis, o que corrobora com o fato de que 20,13\% corresponde ao teor de cinzas das folhas da planta, enquanto o caule apresenta um valor de apenas 5,95\% (ZEM, 2017).

Ainda neste contexto, os teores de cálcio e o sódio aumentaram em 102,04 mg de 5,74 $\mathrm{mg}$, respectivamente no produto formulado com a farinha de ora-pro-nóbis. Zem (2017), em seu trabalho obteve um aumento do cálcio de $212 \mathrm{mg}$ para o cupcake e de $799 \mathrm{mg}$ para o macarrão quando empregou a ora-pro-nóbis, enquanto o teor de sódio variou. No caso do cupcake foi menor em $7 \mathrm{mg}$ e para o macarrão o teor aumentou em $35 \mathrm{mg}$. O destaque neste caso fica para o aumento do teor de cálcio pois, de acordo com Ferreira et al. (2015), a planta ora-pro-nóbis é rica neste mineral, sendo assim capaz de auxiliar na prevenção da osteoporose.

Segundo a Agência Nacional de Vigilância Sanitária (BRASIL, 2012), produtos sólidos, como bolos, podem ser considerados fonte de cálcio quando apresentam mínimo de $15 \%$ da ingestão diária recomendada por $100 \mathrm{~g}$, permitindo que o bolo enriquecido com farinha de ora-pro-nóbis seja considerado fonte de cálcio.

\subsection{Teores de Extrato Etéreo}

O bolo enriquecido com a farinha de ora-pro-nóbis resultou em uma redução de 1,3\% de extrato etéreo em relação ao bolo elaborado apenas com a farinha de arroz, conforme mostrou a Tabela 2. Mundim (2013), em seu trabalho, elaborou um picolé enriquecido com a farinha de ora-pro-nóbis que também apresentou uma redução no teor de extrato etéreo. Esses resultados são diferentes do que foram encontrados nos trabalhos de Rocha et al. (2008), Zem (2017) e Vieira et al. (2019), onde seus produtos enriquecidos com a farinha de ora-pro-nóbis apresentaram maiores teores de extrato etéreo. Os autores acreditam que a diminuição do extrato etéreo também esteja relacionada ao fato da ora-pro-nóbis apresentar um alto teor de fibras (ALMEIDA et al., 2014; ZEM, 2017), que poderiam ter absorvido esse extrato.

Quando avaliamos especificamente as gorduras saturadas e trans nas Tabelas 3 e 4, podemos observar que os teores são semelhantes nos produtos com e sem farinha de ora-pronóbis, o que se dá devido ao fato de que estas estão presentes no recheio que foi idêntico para as duas formulações. 


\subsection{Teores de Proteínas}

Em vários trabalhos é mencionado que a ora-pro-nóbis possui alto teor de proteínas, que varia de $17 \%$ a $29 \%$, justificando o fato de ser popularmente conhecida como "carne de pobre", e destacando a presença dos 9 aminoácidos essências (RODRIGUES et al., 2000; ROCHA et al., 2008; TAKEITI et al., 2009; ALMEIDA ; CORRÊA, 2012; MUNDIM, 2013; ALMEIDA et al., 2014; FERREIRA et al., 2015; MARINELLI, 2016; TRENNEPOHL, 2016; ZEM, 2017; CARVALHO et al., 2019; SATO et al., 2019; VIEIRA et al., 2019).

Ao comparar os teores de proteínas na composição centesimal (Tabela 2) deste trabalho, o bolo enriquecido com a farinha de ora-pro-nóbis resultou em um teor de 0,4\% (composição centesimal) a mais do que no bolo contendo apenas a farinha de arroz. Considerando que os dois produtos foram elaborados a partir dos mesmos ingredientes, constata-se que o aumento ocorreu devido adição da ora-pro-nóbis. Além disso, Zem (2017), elaborou um cupcake com ora-pro-nóbis que teve um aumento de 0,88\% de proteínas, Rocha et al. (2008), elaborou um macarrão com ora-pro-nóbis que teve um acréscimo de 0,78\%, e Vieira et al. (2019), elaborou um cookie que teve um aumento de 3,6\%, o que comprova que ao se adicionar a farinha de ora-pro-nóbis nos produtos alimentícios, realmente ocorre aumento do teor de proteína, que é considerada de boa qualidade, apresentando uma digestibilidade entre $75,9 \%$ e $85,0 \%$ e que contém os 9 aminoácidos essências. Ainda, ressalta-se a isenção de glúten, uma vez que este está presente no trigo, centeio, cevada, malte e aveia (CAMBRAIA, 1980; FERREIRA et al., 2009; TAKEITI et al., 2009; MARINELLI, 2016; TRENNEPOHL, 2016), tornando a ora-pro-nóbis uma opção para pessoas com doença celíaca.

\subsection{Teores de Fibra Alimentar}

A fibra alimentar, segundo Bolzan (2013), representa a soma de todos os polissacarídeos vegetais da dieta, incluindo a celulose, lignina, hemicelulose, pectinas, gomas e mucilagens. Esse constituinte é resistente à ação das enzimas digestivas humanas, podendo reduzir os níveis de pressão arterial, melhorar o controle de glicemia em pacientes com diabete melito, atuar na melhora no sistema imunológico e retardar a digestão e absorção de alguns compostos presentes no organismo (RODRIGUES et al., 2000; BERNAUD ; RODRIGUES, 2013; CARVALHO et al., 2019). 
A farinha de ora-pro-nóbis proporcionou no bolo, conforme a observação dos rótulos nutricionais (Tabelas 3 e 4) a quantidade de 1,0 g a mais de fibra do que no bolo contendo apenas farinha de arroz. Na Tabela 2, o bolo enriquecido com a farinha de ora-pro-nóbis teve um aumento de 2,53\% na fibra alimentar total em comparação com o bolo sem o enriquecimento. Esse aumento se dá devido a utilização da ora-pro-nóbis, e conforme Rodrigues et al. (2000) e Silva ; Pinto (2006), a ora-pro-nóbis é considerada um complemento nutricional por fornecer alto teor de fibra. A afirmação é comprovada também pelos trabalhos de Zem (2017), que elaborou um macarrão que teve um aumento de 4,15\%, Rocha et al. (2008), também elaborou um macarrão que teve um acréscimo de 0,25\% e Vieira et al. (2019), elaborou um cookie que teve um aumento de 15,58\%. Isso ressalta a importância da utilização da farinha de ora-pro-nóbis em novos alimentos, pois suas fibras possuem dois polissacarídeos (arabinogalactanas e galactomanas) que estão associados ao sistema imune e apresentam propriedades de absorção de água, auxiliando no funcionamento do trato digestivo (SEYFRIED, 2016; TONON et al., 2018).

Produtos sólidos, como bolos, podem ser considerados rico em fibra alimentar quando apresentam mínimo de $6 \mathrm{~g}$ de fibra em $100 \mathrm{~g}^{-1}$, tornando o bolo enriquecido com farinha de ora-pro-nóbis um produto rico em fibra alimentar (BRASIL, 2012).

\subsection{Valor energético}

O valor energético é um dado para se avaliar o bom funcionamento do corpo. Alimentos que apresentam um valor energético muito grande são aqueles que contém grandes quantidades de açúcares, óleo e gorduras. Para estabelecer o valor energético de cada alimento, é considerado o calor de combustão e a digestibilidade, de acordo com o sistema Atwater, para os teores de proteína, carboidratos e lipídeos. Para cada parâmetro utiliza-se coeficientes específicos, sendo 4 para proteína, 4 para carboidratos e 9 para lipídeos (Lima et al., 2001; MENDONÇA, 2010; PAWAK ; FERREIRA, 2013; TRENNEPOHL, 2016).

Nas Tabelas 3 e 4, podemos observar que o bolo enriquecido com a farinha de ora-pronóbis apresentou uma diminuição de $9 \mathrm{kcal}$ em relação ao bolo sem a farinha de ora-pronóbis. Essa redução pode ser devido ao fato de o arroz ser um alimento altamente energético (FRANCO et al., 2018) e na receita enriquecida ter sido diminuída a quantidade da farinha de arroz. 
No trabalho de Zem (2017), o macarrão elaborado com farinha de ora-pro-nóbis apresentou uma diminuição de 48,3 kcal, Rocha et al. (2008), também elaborou um macarrão com farinha de ora-pro-nóbis e obteve uma diminuição de 13,65 kcal, Vieira et al. (2019), elaborou um cookie com farinha de ora-pro-nóbis e teve uma redução de 10,73 kcal.

De forma geral, alimentos com menores valores energéticos são de extrema importância para saúde do ser humano, já que atualmente a obesidade e a vida sedentária estão provocando preocupações generalizadas (ZEM, 2017; SATO et al., 2019).

\subsection{Teores de Carboidratos disponíveis}

O carboidrato é importante para os seres humanos, pois ele é uma fonte de energia, age na preservação das proteínas, protege contra corpos cetônicos, é combustível para o sistema nervoso central e é a base da dieta da população (PINHEIRO, PORTO ; MENEZES, 2005; NELSON ; COX, 2014). O bolo não enriquecido com a farinha de ora-pro-nóbis apresentou um teor de 3,87\% a mais de carboidrato na composição centesimal (Tabela 2).

Nos trabalhos de Zem (2017), Rocha et al. (2008) e Vieira et al. (2019), todos os produtos que tiverem adição da farinha de ora-pro-nóbis apresentaram valores menores de carboidratos nos produtos finais, justificando a adição da farinha de ora-pro-nóbis em diferentes tipos de produtos a fim de se obter um produto menos calórico e com alto teor de fibra.

\subsection{Análise estatística}

A análise multivariada de componentes principais (ACP), Figura 2, foi empregada para avaliar a influência dos parâmetros na separação dos produtos com enriquecimento da farinha de ora-pro-nóbis (A1, A2 e A3) e sem o enriquecimento com a farinha de ora-pronóbis (A4, A5 e A6). Observa-se que foram necessários 2 componentes para a separação dos grupos e que os parâmetros que mais influenciaram na separação foram fibra solúvel, fibra insolúvel, cinzas, sódio e cálcio.

Ainda que o teor de extrato etéreo e de proteína não tenha grande influência na separação dos grupos, vale ressaltar que o teor de extrato etéreo tende diminuir nos produtos enriquecidos com a farinha de ora-pro-nóbis o que contribui para um menor valor calórico. 
Figura 2 - Gráfico dos scores das duas primeiras componentes principais para as amostras enriquecidas com a farinha de ora-pro-nóbis (A1, A2 e A3) e para as amostras não enriquecidas (A4, A5 e A6). PC1 explica 64,57\% da variância total dos dados, enquanto a PC2 explica 19,84\%.

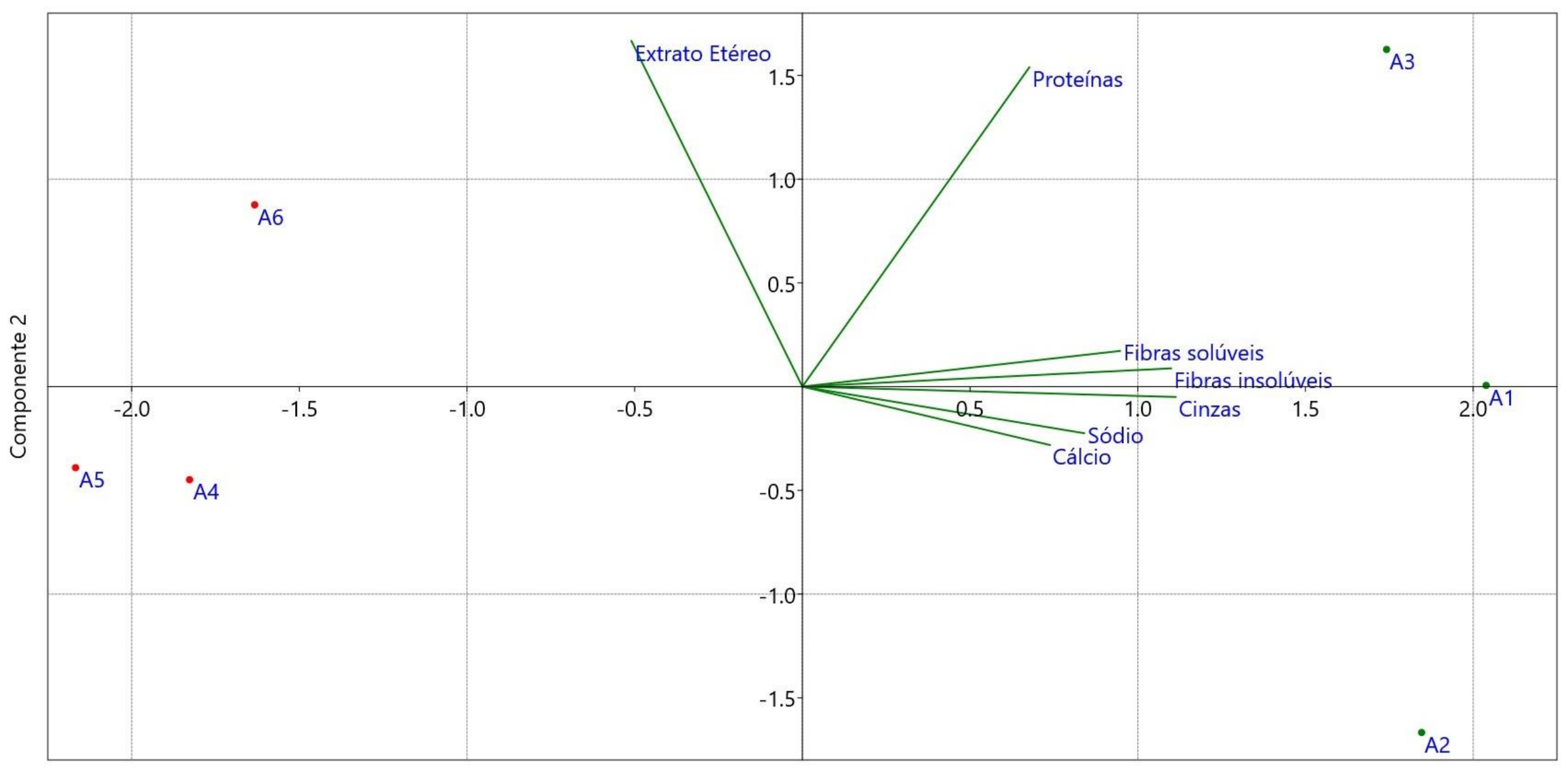

\section{Componente 1}

Fonte: Elaborado pelos Autores. 


\section{CONCLUSÃO}

Por meio desse trabalho, foi possível observar que a adição da farinha de ora-pro-nóbis na formulação de receitas alimentícias, como forma de enriquecimento, é viável, pois tem alto valor nutritivo, já que apresenta bons teores de cálcio, fibra alimentar e proteínas, a qual é benéfica à saúde, considerada uma alternativa para aqueles que tem restrições alimentares, como é o caso do glúten. Os bolos enriquecidos com a farinha de ora-pro-nóbis liofilizada apresentaram aparência esverdeada seca, devido a quantidade de pigmentos característicos da planta.

\section{REFERÊNCIAS}

ALMEIDA, M. E. F. de; CORRÊA, A. D. Utilização de cactáceas do gênero Pereskia na alimentação humana em um município de Minas Gerais. Ciência Rural, Santa Maria, v.42, n.4, p. $751-756,2012$

ALMEIDA, M. E. F. de et al. Caracterização química das hortaliças não-convencionais conhecidas como ora-pro-nóbis. Biosci. J., v. 30, supplement 1, p. 431-439, 2014.

ASP, N. G. et al. Rapid enzymatic assay of insoluble and soluble dietary fiber. Journal of Agricultural and Food Chemistry, Bethesda, v. 31, n. 3, p. 476-482, 1983.

BERNAUD, F. S.; RODRIGUES, T. C. Fibra alimentar - Ingestão adequada e feitos sobre a saúde do metabolismo. Archives of Endocrinology and Metabolism, v. 57, n. 6, p. 397-405, 2013.

BOLZAN, R. C. Bromatologia. Colégio Agrícola de Frederico Westphalen-RS, 2013.

BORGES, J. T. da S. et al. Caracterização Físico-Química e sensorial de pão de sal enriquecido com farinha integral de linhaça. Boletim do Centro de Pesquisa de Processamento de Alimentos, Curitiba, v. 29, n. 1, p. 83-96, jan./jun. 2011.

BRASIL, Agência Nacional de Vigilância Sanitária. RDC nº 359, de 23 de dezembro de 2003. Aprova Regulamento técnico de porções de alimentos embalados para fins de rotulagem nutricional. Diário Oficial da União, Brasília, DF, 26 dez. 2003 a.

BRASIL, Agência Nacional de Vigilância Sanitária. RDC nº 360, de 23 de dezembro de 2003. Aprova o Regulamento Técnico sobre Rotulagem Nutricional de Alimentos Embalados, tornando obrigatória a rotulagem nutricional. Diário Oficial da União, Brasília, DF, 26 dez. 2003b.

BRASIL, Agência Nacional de Vigilância Sanitária. RDC no 54, de 12 de novembro de 2012. Dispõe sobre o Regulamento Técnico sobre Informação Nutricional Complementar. Diário Oficial da União, Brasília, DF, 13 nov. 2012.

CAMBRAIA, J. Valor nutricional do ora-pro-nóbis. Viçosa, Universidade Federal de Viçosa, p. 2, 1980. (Informe Técnico). 
CARVALHO, M. F. F. et al. Centesimal Evaluation of Two Species of Ora-pro-nobis (Pereskia aculeata Miller and Pereskia grandifolia Haw) and Application in Extruded Product. Chemical Engineering Transactions, v. 75, p. 325-330, 2019.

DORS, G. C.; PINTO, R. H.; BADIALE-FURLONG, E. Influência das condições de parboilização na composição química do arroz. Ciênc. Tecnol. Aliment., v. 29, n. 1, Campinas, 2009.

FAO. Relatório Global de Crises Alimentares 2019: análise conjunta para melhores decisões. 2019. Disponível em: < http://www.fsinplatform.org/sites/default/files/resources/files/GRFC\%202019_Full\%20Report.pd f>. Acesso em: 26 jun. 2019.

FASANO, A. et al. Federation of International Societies of Pediatric Gastroenterology, Hepatology, and Nutrition Consensus Report on Celiac Disease. Journal of Pediatric Gastroenterology and Nutrition, v. 47, n. 2, p. 214-219, 2008.

FERREIRA, L.C. et al. Análise de ferro e cálcio presente na planta Pereskia aculeata Miller. In: ENCONTRO NACIONAL DE TECNOLOGIA QUÍMICA, 8.[Anais...]. Vitória/ES, 9 -11 set. 2015.

FERREIRA, S. M. R. et al. Cookies sem glúten a partir da farinha de sorgo. Archivos Latinoamericanos de Nutrición, v. 59, n. 4, p. 433-440, 2009.

FRANCO, V. A. et al. Propriedades reológicas e composição proximal da farinha de arroz e farinha de batata-doce. Científic@ - Multidisciplinary Journal. V. 5, n. 3, p. 113-124, 2018.

GALLAGHER, E.; GORMLEY, T. R.; ARENDT, E. K. Recent advances in the formulation of gluten-free cereal-based products. Trends in Food Science ; Technology, v.15, n. 3-4, p.143$152,2004$.

HAMMER, Ø.; HARPER, D. A. T.; RYAN, P. D. PAST: Paleontological Statistics Software Package For Education And Data Analysis. Palaeontologia Electronica, v. 4, n. 1, p. 9. 2001.

HONGYU, K.; SANDANIELO, V. L. M.; OLIVEIRA JUNIOR, G. J. de. Análise de Componentes Principais: resumo teórico, aplicação e interpretação. $E ; S$ - Engineering and Science, v. 1, ed. 5, 2016. Disponível: <10.18607/ES20165053>. Acesso em: 05 mar. 2020.

INSTITUTO ADOLFO LUTZ. Métodos físico-químicos para análise de alimentos. São Paulo: Instituto Adolfo Lutz, p. 1020, ed. 4. 2008.

MARINELLI, P. S. Farinhas de moringa (Moringa Oleifera Lam.) e ora-pro-nóbis (Pereskia aculeata Mill.): Biomateriais funcionais. Tese (Doutorado em Ciência e Tecnologia de Materiais) Programa de Pós-Graduação em Ciência e Tecnologia de Materiais, Universidade Estadual Paulista "Júlio de Mesquita Filho", Bauru, f. 59, 2016.

MENDONÇA, S. N. T. G. Nutrição. Curitiba: Editora do Livro Técnico, v. 1. p. 128, 2010.

MORETTO, E. et al. Introdução à ciência de alimentos. Florianópolis: Editora da UFSC, 2002. $255 \mathrm{p}$. 
MUNDIM, M. S. Estudo da composição centesimal e teste de aceitabilidade de picolés de limão com e sem ora-pro-nóbis (Pereskia aculeata Miller). Trabalho de Conclusão de Curso (Graduação em Nutrição) - Universidade Federal de Uberlândia, 2013.

NASCIMENTO, A. B. Desenvolvimento de produto alimentício sem glúten elaborado a partir de percepções de consumidores celíacos. Universidade Federal de Santa CatarinaCentro de Ciências Agrarias. Florianopolis-SC, 2014.

NELSON, D. L.; COX, M. M. Princípios de Bioquímica de Lehninger. 6. ed. Porto Alegre: Artmed, 2014.

OLIVEIRA, J. L. de. Pães sem glúten com farinha de sorgo vermelho: propriedades tecnológicas, proximais e sensoriais. Dissertação (Mestrado em Tecnologia de Alimentos) Programa de Pós-Graduação em Tecnologia de Alimentos, Instituto Federal Goiano, Rio Verde, f. 81, 2017.

PAWAK, T. I. A. S. FERREIRA, M. A. O. A importância de conhecer o valor nutricional dos alimentos através dos rótulos das embalagens. In: OS DEFEITOS da escola pública paranaense na perspectiva do professor PDE. Universidade Estadual do Norte do Paraná-PR, 2013.

PINHEIRO, D. M.; PORTO, K. R. de A.; MENEZES, M. E. da S. A química dos alimentos: carboidratos, lipídios, proteínas e minerais. Maceió: EDUFAL, 2005.

ROCHA, D. R. C. et al. Macarrão adicionado de ora-pro-nóbis (Pereskia aculeata Miller) desidratado. Alimentos e Nutrição, v. 19, n. 4, p. 459-465, 2008.

RODRIGUES, S. et al. Caracterização química e nutricional da farinha de ora-pro-nóbis (Pereskia aculeata Mill.). Centro Estadual de Educação Tecnológica Paulo Souza. Faculdade de Tecnologia Estudante Rafael Almeida Camarinha. Marilia-SP. 2000.

SATO, R. et al. Nutritional improvement of pasta with Pereskia aculeata Miller: a nonconventional edible vegetable. Food Science and Technology, Campinas, 39 (Suppl. 1): 28-34, June 2019.

SEYFRIED, M. Pectinas de plantas medicinais: características estruturais e atividades imunomoduladoras. Rev. Bras. Pl. Med., Campinas, v.18, n.1, p.201-214, 2016.

SILVA, M. C. et al. Teores de proteínas e fibras das folhas de taioba, ora-pro-nóbis, serralha e mostarda coletadas no município de Diamantina. In SEMINÁRIO (ED.), DE PESQUISA E EXTENSÃO, 7. [Anais...] Diamantina MG, Brasil: UEMG.

TACO Tabela brasileira de composição de alimentos / NEPA - UNICAMP.- 4. ed. rev. e ampl.. Campinas: NEPA- UNICAMP, 2011. 161 p.

TAKEITI, C. Y. et al. Nutritive evaluation of a non-conventional leafy vegetable (Pereskia aculeata Miller). International Journal of Food Sciences and Nutrition, v. 60, p. 148-160, 2009.

TONON, R. V. et al. Tecnologias para o Aproveitamento Integral dos Resíduos da Indústria Vitivinícola. Rio de Janeiro: Embrapa Agroindústria de Alimentos, p. 41, 2018. 
TRENNEPOHL, B. I. Caracterização físico-química, atividade antioxidante e atividades biológicas da espécie Pereskia aculeata Mill. Dissertação (Mestrado em Alimentação e Nutrição) Programa de Pós-Graduação em Alimentação e Nutrição, área de concentração Qualidade dos Alimentos e Nutrição, setor de Ciências da Saúde, Universidade Federal do Paraná, Curitiba, f. 97, 2016.

VIEIRA, C. R. et al. Effect of Pereskia aculeata Mill. in vitro and in overweight humans: A randomized controlled trial. Journal of Food Biochemistry, v. 43, n. 7, p. 1-10, 2019.

VIZZOTO, T. et al. Determinação de umidade e cinzas em diversas matrizes alimentares. Instituto Federal Catarinense- Campus Concórdia- SC. 2010.

ZEM, L. M. Pereskia aculeata Mill: propagação vegetativa, análise anatômica, bromatológica e biológica. Tese (Doutorado em Ciências) Programa de Pós-Graduação em Agronomia, Área de Concentração em Produção Vegetal, Departamento de Fitotecnia e Fitossanitarismo, Setor de Ciências Agrárias, Universidade Federal do Paraná, Curitiba, f. 233, 2017. 